\title{
Adaptive Relay Selection Scheme Based on Bayesian Inference (AS-BI) for Cooperative Communication in 5G Networks
}

\author{
Madeeha Ishtiaq ${ }^{1}$, Shakeel A. Waqas ${ }^{2}$, Muhammad Saifullah ${ }^{3}$, Nazih K. Mallat ${ }^{4}$ \\ ${ }^{1}$ Department of Computer Science, Women University Swabi, Pakistan \\ ${ }^{2}$ University of Engineering and Technology Mardan, Pakistan \\ ${ }^{3}$ National University of Science and Technology, Pakistan \\ ${ }^{4}$ College of Engineering, Al Ain University, United Arab Emirates
}

\begin{abstract}
In this paper, a Bayesian inference-based relay selection scheme, also called Adaptive Selection on Bayesian Inference (AS-BI) for cooperative networks in 5G networks is invoked. This scheme works on the principle of Amplify-and-Forward (AF) protocol which selects the most optimal relay towards the destination. This paper proposed a probabilitybased relay selection scheme in which posterior probability is calculated for the selection of relay node based on the prior and conditional probabilities. The proposed scheme has been accepted as optimal solution for relay selection, which significantly enhances the network performance by reducing (BER) for constant SNR.
\end{abstract}

Keywords - Cooperative Network, 5G, Amplify-andForward, relay selection scheme, Bayesian Inference.

\section{Introduction}

The 5G communication has embarked to operate with very high frequencies. However, the signaling range of communication becomes shorter.

DOI: 10.18421/TEM102-43

https://doi.org/10.18421/TEM102-43

Corresponding author: Nazih K. Mallat, College of Engineering, Al Ain University, United Arab Emirates.

Email: nazih.mallat@aau.ac.ae

Received: 09 January 2021.

Revised: 06 April 2021.

Accepted: 12 April 2021.

Published: 27 May 2021.

(cc) BY-NC-ND (C) 2021 Madeeha Ishtiaq et al; published by UIKTEN. This work is licensed under the Creative Commons Attribution-NonCommercial-NoDerivs 4.0 License.

The article is published with Open Access at www.temjournal.com
In order to provide reliable communication, the cooperative communication earned a lot of attention due to its potential applications which provide cooperation between the end nodes. Moreover, due to the enormous growth of data-rate applications, it witnessed a shortage of spectrum band. In this regard, a resource efficient communication has been paid attention due to the limited resource and environment friendly transmission behaviors. The cooperation between the nodes has been invoked to utilize the resources efficiently and enhances the capacity [1].

The cooperative communication demonstrates that single antenna in a multi-user state share their antennas to make a virtual multiple-antenna transmitter which enables them to accomplish transmit diversity [2]. In the case of relay-based systems, it is very challenging how to use relay nodes effectively and how to achieve maximum performance of the systems. In this regard, different techniques have been studied by the research and academia to optimize the network performance.

Furthermore, the probability-based relay selection scheme has been proposed using Bayesian theory to calculate the probability of selecting a relay node based on the achievable data rate. The achievable data rate is quantified as a prior knowledge and posterior probability of selecting a relay node, which maximize the data rate of the network [3]. Moreover, the Multiple---Input Multiple---Output (MIMO) techniques have been enabled to improve capacity i.e., spectral-efficiency (SE), and power consumption. However, more circuit energy is required due to the doubling-up of transmitter and receiver antennas [4]. In [5] [6], regarding to the channel state information, the relay selection scheme using power control is applied based on the defined threshold. Moreover, the authors proposed a scheme relay selection-power allocation-particle Swarm Ant colony optimization (RS-PA-PSACO) based on the PSACO algorithm which may simultaneously obtain 
the optimal solution of relay selection and power allocation to minimize the symbol error rate (SER) with a fixed total power constraint [7], [8]. Further, in [9] the channel state information (CSI) has invoked as some selection criteria with location of relays and the distance between them which significantly improves the SNR and reduces the BER. According to the CSI, the SNR can be maximized by adjusting the transmission power of the selected relay. This scheme increases the received SNR and lower BER as compared to the simple average power allocation scheme [10], [11]. The Energy Efficiency is widely accepted for cooperative networks. In order to increase the energy efficiency, an auction-based model is proposed. Hence source will send the data with minimum transmission power and relay may also have consumed minimum energy, which significantly increases energy efficiency of the whole network [12]. Moreover, a threshold-based relay selection scheme has been proposed also known as Optimal-threshold multiple---relay--selection (OT-MRS). These relays have selected where SNR of the maximal ratio combines and direct path exceeds a threshold value, this scheme provides special diversity and efficient utilization of bandwidth [13]. To increase the data rate of the source node, an optimal polynomial time algorithm is proposed for the relay assignment by which firstly all the sources will be assigned relay randomly. Similarly, the iteration algorithm identifies the source node which has minimum data rate which helps this node to find another relay so that its data rate can be increased [14].

Moreover, another threshold-based relay selection scheme is proposed in which reliable appropriate relays are determined by comparing source to relay SNR with a threshold value, and one of the reliable relays is selected by the destination based on relay destination SNR. This technique eliminates the concept of SNR of source to relay be known at the destination and it is replaced by a threshold value and the threshold increases logarithmically with the increase of SNR of source to relay links and linearly with number of relays [15], [16]. To enhance the energy efficiency of wireless networks, fixed relay nodes can be utilized. A properly arrange fixed relay nodes can adequately take care of path loss [17]. More precisely, [18], [19], [20] has studied that selected relay holds the substantial end-to-end relaying path. It achieves optimal performance and ensures a full diversity equal to the number of relays. Moreover, [21], [22] has studied the cooperative diversity performance by selecting the best relay according to the average SNR, and outage probability selection of relay according to the instantaneous SNR. However, relay selection in cooperative communication is a major problem. In this regard, [23] has proposed an algorithm, such that total capacity is maximized among all possible relay assignments. Furthermore, [24], [25], [26], has discussed both joint relay node selection and power allocation with the aim to minimize the total power consumption of the network while utilizing bandwidth efficiently. In [27], [28], [29], authors presented four types of different relay selection schemes such as energy utilization, maximizing capacity scheme (MCS), energy prior scheme (EPS), mixed energy-capacity-scheme (MECS), and weighted ECS (WECS). More, [30] has invoked a power allocation scheme for cooperative communication with decode-and-forward (DF) protocol for source and relays nodes to optimize the power consumption, while retain the quality of services. Furthermore, [31], [32], [33], [34] has studied a dispersed relay selection scheme, where one client selects the best end-to-end route among numerous relays in view of momentary channel estimations.

After this introduction, in Section 2 we explain the system model for the cooperative communication in multi-hop cellular network. The Section 3 discusses the Bayesian inference, relay selection scheme and selection criteria that how relays are selected using AS-BI, for the transmission with the help of flow chart. Then, the Section 4 explains the simulation model and simulation results. Finally, the paper is concluded by the future work.

\section{System Model}

The proposed system model has shown in Figure 1, which is based on a source (S) and relays (R). The source may be a base station or users with packets, waiting for transmission in cellular network whereas, the relays are considered as user equipment (UE) at both sending and a destination side.

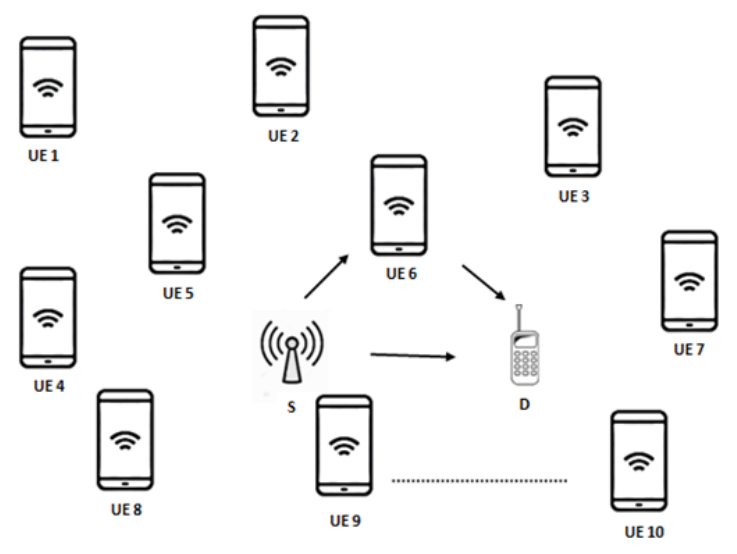

Figure 1. Cooperative Communication in cellular Network

Furthermore, the model acknowledges communication between source and destination through the UE and the selection of UE is based on their battery power level. 
Here communication can take place by two paths, through direct path and with the help of relay or both. When communication take place through direct path then received signal $Y_{S \rightarrow D}$ can be given as.

$$
Y_{S \rightarrow D}=h_{S \rightarrow D} x+N_{S \rightarrow D}
$$

In which $h_{S \rightarrow D}$ is the channel state information from source to destination, $x$ is the transmitted signal while $N_{S \rightarrow D}$ is the noise of the channel from source to destination.

When transmission take place with the help of relay then transmitted signal received at the destination in two stages.

In stage 1, signal is transmitted form source to the relay and received signal at the relay $Y_{S \rightarrow R}$ can be given as.

$$
Y_{S \rightarrow R}=h_{S \rightarrow R} x+N_{S \rightarrow R}
$$

In which $h_{S \rightarrow R}$ is the channel state information from source to destination, $x$ is the transmitted signal, and $N_{S \rightarrow R}$ is the channel noise from source to relay.

In stage 2, signal is transmitted from relay to destination using AF protocol and signal received at the destination $Y_{R \rightarrow D}$ will be given as.

$$
Y_{R \rightarrow D}=\alpha h_{R \rightarrow D} Y_{S \rightarrow R}+N_{R \rightarrow D}
$$

In which $h_{R \rightarrow D}$ is the channel state information from relay to destination, $\alpha$ is the amplification coefficient, $Y_{S \rightarrow R}$ is the signal received at the relay, and $N_{R \rightarrow D}$ is the noise of the channel from relay to destination. We calculated SNR for AF protocol through relay path ( $S$ to $R \& R$ to $D$ ) and through direct path ( $\mathrm{S}$ to $\mathrm{D}$ ) which is given by the authors of [16].

$$
S N R_{S \rightarrow R} \& R \rightarrow D=\frac{g \alpha_{1} \alpha_{2} P_{T}}{g \alpha_{2} N_{1}+N_{2}}
$$

while,

$$
S N R_{S \rightarrow D}=\frac{\alpha_{d} P_{T}}{N_{2}}
$$

Where $g$ is the amplification gain, while $\alpha_{1}, \alpha_{2}, \alpha_{d}$ is channel state information from $\mathrm{S}$ to $\mathrm{R}, \mathrm{R}$ to $\mathrm{D}$, and $\mathrm{S}$ to $\mathrm{D}$ respectively and $N_{1}, N_{2}$ are the relay receiver and destination receiver noises, respectively. Then we calculate $C G$ of the relay path ( $S$ to $R \& R$ to $D$ ) and direct path ( $\mathrm{S}$ to $\mathrm{D}$ ) which will be given as

$$
C G_{S \rightarrow D}=\frac{\epsilon_{o}}{\beta_{o}}
$$

while,

$$
C G_{S \rightarrow R \& R \rightarrow D}=\frac{\epsilon_{1} / \beta_{o}}{\epsilon_{o}}
$$

In which $\epsilon_{o}, \epsilon_{1}$ is the error in number of bits transmitted, through direct path and through relay path, respectively. While $\beta_{o}$ is the total number of bits generated from source. Then we calculate $C H G$ of the relay path ( $S$ to $R \& R$ to $D$ ) and direct path ( $S$ to $\mathrm{D})$ which will be given as

$$
\begin{aligned}
& C H G_{S \rightarrow R \& R \rightarrow D} \\
& =\operatorname{abs}\left(\min \left(\operatorname{mean}\left(\alpha_{1}\right), \text { mean }\left(\alpha_{2}\right)\right)\right)
\end{aligned}
$$

while,

$$
\begin{aligned}
& C H G_{S \rightarrow D} \\
& =\operatorname{abs}\left(\operatorname{mean}\left(\alpha_{d}\right)\right)
\end{aligned}
$$

\section{Bayesian Inference \& Relay Selection Scheme}

In this section, the probabilities calculation and the proposed scheme of adaptive selection based on Bayesian inference are presented.

\subsection{Bayesian inference \& Probabilities Calculation}

Bayesian inference is statistical approach to the problem formulation. Bayesian inference derives posterior probability of the problem from the two already known probabilities which is prior probability and conditional probability. Prior probability shows the estimate of current evidence collected about the problem or it reflects our prior knowledge which will be given as $\mathrm{P}\left(\mathrm{S}_{\mathrm{n}}\right)$. While conditional probability shows the estimate of occurrence of some unknown variable $(\mathrm{X})$ based on our observed evidence and will be given as $\mathrm{P}\left(\mathrm{X} \mid \mathrm{S}_{\mathrm{n}}\right)$. When both the prior and conditional probability are known posterior probability for $\mathrm{N}$ number of relay nodes can be given as.

$$
P\left(S_{n} \mid X\right)=\frac{P\left(X \mid S_{n}\right) P\left(S_{n}\right)}{\sum_{n=1}^{N} P\left(X \mid S_{n}\right) P\left(S_{n}\right)}
$$

Source node will select a relay node with the help of above posterior probability. When SNR, CG and $\mathrm{CHG}$ of direct and relay path are known then prior probability of source node $\left(S_{n}\right)$ will be given as: 


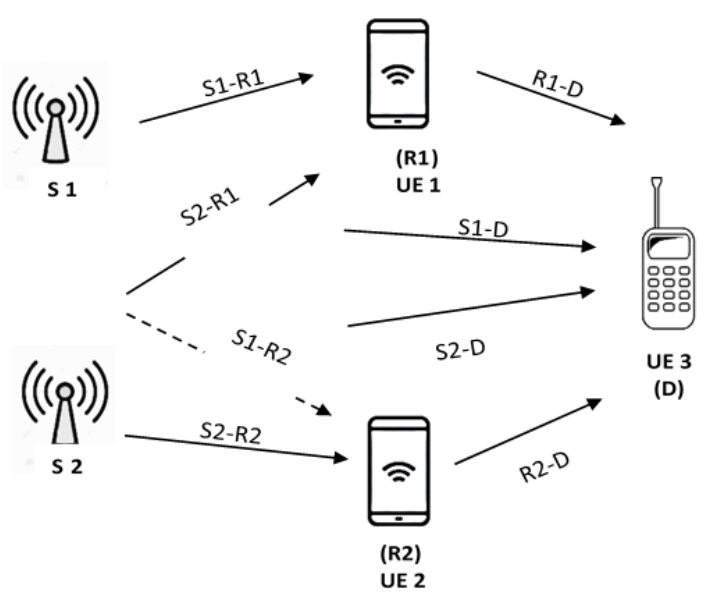

Figure 1. Simulation model for AS-BI Relay Selection

$$
\begin{gathered}
\mathrm{P}_{\mathrm{SNR}}\left(\mathrm{S}_{\mathrm{n}}\right)=\frac{\mathrm{SNR}_{\mathrm{S} \rightarrow \mathrm{D}_{\mathrm{n}}}}{\sum_{\mathrm{t}=1}^{\mathrm{N}_{\mathrm{s}} \mathrm{SNR}_{\mathrm{S} \rightarrow \mathrm{D}_{\mathrm{t}}}}} \\
\mathrm{P}_{\mathrm{CG}}\left(\mathrm{S}_{\mathrm{n}}\right)=\frac{\mathrm{CG}_{\mathrm{S} \rightarrow \mathrm{D}_{\mathrm{n}}}}{\sum_{\mathrm{t}=1}^{\mathrm{N}_{\mathrm{s}} \mathrm{CG}_{\mathrm{S} \rightarrow \mathrm{D}_{\mathrm{t}}}}} \\
\mathrm{P}_{\mathrm{CHG}}\left(\mathrm{S}_{\mathrm{n}}\right)=\frac{\mathrm{CHG}_{\mathrm{S} \rightarrow \mathrm{D}_{\mathrm{n}}}}{\sum_{\mathrm{t}=1}^{\mathrm{N}_{\mathrm{s}}} \mathrm{CHG}_{\mathrm{S} \rightarrow \mathrm{D}_{\mathrm{t}}}}
\end{gathered}
$$

Where $N_{s}$ is the number of sources while conditional probability of a relay node $\left(R_{m}\right)$ will be given as

$$
\begin{aligned}
& \mathrm{P}_{\mathrm{SNR}}\left(\mathrm{R}_{\mathrm{m}} \mid \mathrm{S}_{\mathrm{n}}\right)=\frac{\mathrm{SNR}_{\mathrm{S} \rightarrow \mathrm{R} \& R \rightarrow D_{\mathrm{mn}}}}{\sum_{\mathrm{n}=1}^{\mathrm{N}_{\mathrm{r}}} \mathrm{SNR}_{\mathrm{S} \rightarrow \mathrm{R} \& R \rightarrow D \mathrm{mn}}} \\
& \mathrm{P}_{\mathrm{CG}}\left(\mathrm{R}_{\mathrm{m}} \mid \mathrm{S}_{\mathrm{n}}\right)=\frac{\mathrm{CG}_{\mathrm{S} \rightarrow \mathrm{R} \& R \rightarrow D \mathrm{mn}}}{\sum_{\mathrm{n}=1}^{\mathrm{N}_{\mathrm{r}}} \mathrm{CG}_{\mathrm{S} \rightarrow \mathrm{R} \& R \rightarrow D \mathrm{mn}}} \\
& \mathrm{P}_{\mathrm{CHG}}\left(\mathrm{R}_{\mathrm{m}} \mid \mathrm{S}_{\mathrm{n}}\right)=\frac{\mathrm{CHG}_{\mathrm{S} \rightarrow \mathrm{R} \& R \rightarrow D_{\mathrm{mn}}}}{\sum_{\mathrm{n}=1}^{\mathrm{N}_{\mathrm{r}}} \mathrm{CHG}_{\mathrm{S} \rightarrow \mathrm{R} \& R \rightarrow D_{\mathrm{mn}}}}
\end{aligned}
$$

Where $\mathrm{N}_{\mathrm{r}}$ show total number of relays available. According to Bayesian rule we can formulate posterior probability which will be given as

$$
\begin{gathered}
=\frac{P_{S N R}\left(R_{m} \mid S_{n}\right) P_{S N R}\left(S_{n}\right)}{\sum_{n=1}^{N_{r}} P_{S N R}\left(R_{m} \mid S_{n}\right) P_{S N R}\left(S_{n}\right)} \\
=\frac{P_{C G}\left(R_{m} \mid S_{n}\right) P_{C G}\left(S_{n}\right)}{\sum_{n=1}^{N_{r}} P_{C G}\left(R_{m} \mid S_{n}\right) P_{C G}\left(S_{n}\right)}
\end{gathered}
$$

$$
\begin{aligned}
& \mathrm{P}_{\mathrm{CHG}}\left(\mathrm{S}_{\mathrm{n}} \mid \mathrm{R}_{\mathrm{m}}\right) \\
& =\frac{\mathrm{P}_{\mathrm{CHG}}\left(\mathrm{R}_{\mathrm{m}} \mid \mathrm{S}_{\mathrm{n}}\right) \mathrm{P}_{\mathrm{CHG}}\left(\mathrm{S}_{\mathrm{n}}\right)}{\sum_{\mathrm{n}=1}^{\mathrm{N}_{\mathrm{r}}} \mathrm{P}_{\mathrm{CHG}}\left(\mathrm{R}_{\mathrm{m}} \mid \mathrm{S}_{\mathrm{n}}\right) \mathrm{P}_{\mathrm{CHG}}\left(\mathrm{S}_{\mathrm{n}}\right)}
\end{aligned}
$$

After calculating posterior probabilities for all factors, we calculate mean of posterior probability for each factor of same path. Then we will get the probability of a source node that relay $R_{m}$ will be selected. We will assign a relay node when:

$$
\mathrm{P}\left(\mathrm{S}_{\mathrm{n}} \mid \mathrm{R}_{\mathrm{m}}\right)>P\left(\mathrm{~S}_{\mathrm{n}} \mid \mathrm{R}_{\mathrm{k}}\right) \text { forall } \mathrm{m} \neq \mathrm{k}
$$

\subsection{Relay Selection Scheme}

Bayesian inference derives posterior probability upon some prior knowledge. In AS-BI relay selection scheme we first calculated the factors upon which we derive prior probabilities. We calculated SNR, CHG, and $\mathrm{CG}$ for the direct and relay path then we derived prior probability for each source node $\mathrm{P}\left(\mathrm{S}_{\mathrm{n}}\right)$ according to equation $11,12 \& 13$ and conditional probability $\mathrm{P}\left(\mathrm{X} \mid \mathrm{S}_{\mathrm{n}}\right)$ for each relay node according to equation $14,15 \& 16$. Then at last source node calculates the posterior probability of selecting a relay node according to equation 17, 18 and 19 and then selects a relay which has maximum probability value according to rule (20). Fig. 3 shows the flow chart for AS-BI scheme.

\section{Simulation and Discussions}

This section explains the simulation model and simulation results of our proposed AS-BI scheme in which BER is analyzed against fixed SNR value. Analysis is done using AF protocol and Rayleigh fading channel over the system model. In the AS-BI scheme, the channel conditions are the basis of selecting a relay for cooperation, in which the first we determined are SNR, CHG, and CG as well.

\subsection{Simulation Model}

In our simulation model, relay selection scheme is used as shown in Figure 2, in which two sources S1 and S2 are defined and provided with two relays nodes R1\& R2, which are considered as a UE. Source can communicate with destination using one of these relays and through direct path. Two relays are examined for simplicity. Both sources have three available paths for the transmission, the first is the direct path which are S1-D and S2-D while the second is the relay path which are S1-R1,S1 $\mathrm{R} 2, \mathrm{~S} 2-\mathrm{R} 1$ and $\mathrm{S} 2-\mathrm{R} 2$. Each source has two available relays from which it selects one based on posterior probability. On the basis of posterior probability, a single relay serves either both sources, or a single source. 


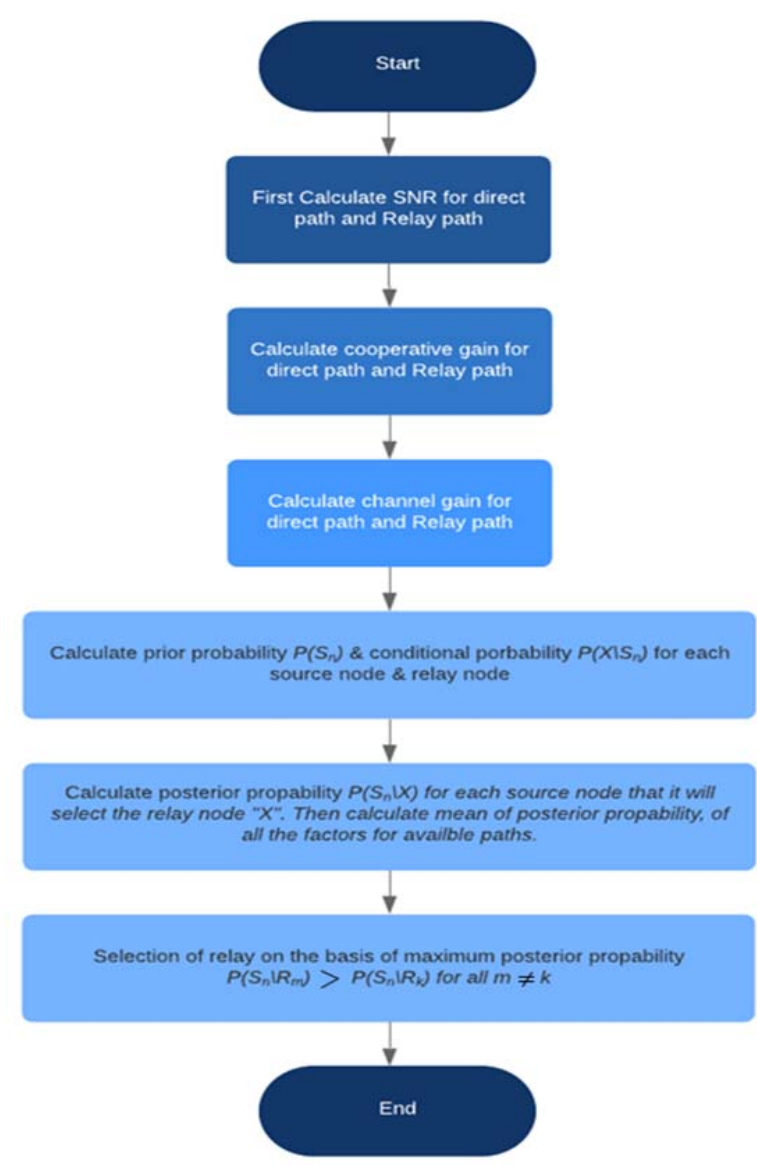

Figure 2. Flow chart of relay selection scheme based on AS-BI

According to simulation model, our network consists of two sources $S 1$ and $S 2$ and two relays $R 1$ and $R 2$. The AF relaying protocol is invoked using Raleigh fading channel. The Table 1 show SNR between both sources and relays. Table 2 depicts cooperative gain for both sources and relays. Table 3 shows channel gain for both sources and relays. Table 4, 5, and 6 show prior probabilities calculated for each factor while Table 7,8 and 9 illustrate posterior probabilities for relay selection.

Table 1. SNR for Direct and Relay Path

\begin{tabular}{|c|c|c|c|}
\hline Sources & SNR $_{\mathrm{S} \rightarrow \mathrm{D}}$ & $\mathrm{R} 1$ & $\mathrm{R} 2$ \\
\hline $\mathrm{S} 1$ & 0.0013 & 0.0004 & 0.0005 \\
\hline S2 & 0.0011 & 0.0007 & 0.0008 \\
\hline
\end{tabular}

Table 1. CG for Direct and Relay Path

\begin{tabular}{|c|c|c|c|}
\hline Sources & $\mathrm{CG}_{\mathrm{S} \rightarrow \mathrm{D}}$ & $\mathrm{R} 1$ & $\mathrm{R} 2$ \\
\hline $\mathrm{S} 1$ & 0.16 & 0.65 & 0.56 \\
\hline $\mathrm{S} 2$ & 0.14 & 0.44 & 0.72 \\
\hline
\end{tabular}

Table 2. CHG for Direct and Relay Path

\begin{tabular}{|c|c|c|c|}
\hline Sources & $\mathrm{CHG}_{\mathrm{S} \rightarrow \mathrm{D}}$ & $\mathrm{R} 1$ & $\mathrm{R} 2$ \\
\hline $\mathrm{S} 1$ & 0.79 & 0.0005 & 0.0001 \\
\hline $\mathrm{S} 2$ & 0.88 & 0.0006 & 0.002 \\
\hline
\end{tabular}

Prior probabilities considering different factor for $\mathrm{S} 1$ and $\mathrm{S} 2$ will be calculated as $\mathrm{P}_{\mathrm{SNR}}\left(\mathrm{S}_{1}\right)=$ $0.0013 / 0.0013+0.0011 \quad$ and $P_{S N R}\left(S_{2}\right)=$ $0.0011 / 0.0013+0.0011$, so that $P_{S N R}\left(S_{1}\right)=0.54$ and $P_{S N R}\left(S_{2}\right)=0.46$ and total probability will be $\mathrm{P}_{\mathrm{SNR}}\left(\mathrm{S}_{1}\right)+\mathrm{P}_{\mathrm{SNR}}\left(\mathrm{S}_{2}\right)=1$. Similarly, $\mathrm{P}_{\mathrm{CG}}\left(\mathrm{S}_{1}\right)=$ $0.16 / 0.16+0.14$ and $P_{C G}\left(S_{2}\right)=0.14 / 0.16+0.14$, so that $\mathrm{P}_{\mathrm{CG}}\left(\mathrm{S}_{1}\right)=0.53 \quad$ andP $\mathrm{CG}_{\mathrm{CG}}\left(\mathrm{S}_{2}\right)=0.47$. $\operatorname{AndP}_{\mathrm{CHG}}\left(\mathrm{S}_{1}\right)=0.79 / 0.79+0.88$ and $\mathrm{P}_{\mathrm{CHG}}\left(\mathrm{S}_{2}\right)=$ $0.88 / 0.79+0.88$, so that $P_{C H G}\left(S_{1}\right)=0.47$ andP $\mathrm{CHG}_{\mathrm{CHG}}\left(\mathrm{S}_{2}\right)=0.53$.

Table 3. Prior Probabilities of SNR for Direct and Relay Path

\begin{tabular}{|c|c|c|c|}
\hline Sources & $\mathrm{P}_{\mathrm{SNR}}\left(\mathrm{S}_{\mathrm{n}}\right)$ & $\mathrm{P}_{\mathrm{SNR}}\left(\mathrm{R}_{1} \mid \mathrm{S}_{\mathrm{n}}\right)$ & $\mathrm{P}_{\mathrm{SNR}}\left(\mathrm{R}_{2} \mid \mathrm{S}_{\mathrm{n}}\right)$ \\
\hline $\mathrm{S} 1$ & 0.54 & 0.36 & 0.38 \\
\hline $\mathrm{S} 2$ & 0.46 & 0.67 & 0.62 \\
\hline
\end{tabular}

Table 4. Prior Probabilities of CG for Direct and Relay Path

\begin{tabular}{|c|c|c|c|}
\hline Sources & $\mathrm{P}_{\mathrm{CG}}\left(\mathrm{S}_{\mathrm{n}}\right)$ & $\mathrm{P}_{\mathrm{CG}}\left(\mathrm{R}_{1} \mid \mathrm{S}_{\mathrm{n}}\right)$ & $\mathrm{P}_{\mathrm{CG}}\left(\mathrm{R}_{2} \mid \mathrm{S}_{\mathrm{n}}\right)$ \\
\hline $\mathrm{S} 1$ & 0.53 & 0.6 & 0.44 \\
\hline $\mathrm{S} 2$ & 0.47 & 0.4 & 0.56 \\
\hline
\end{tabular}

Table 5. Prior Probabilities of CHG for Direct and Relay Path

\begin{tabular}{|c|c|c|c|}
\hline Sources & $P_{C H G}\left(S_{n}\right)$ & $P_{C H G}\left(R_{1} \mid S_{n}\right)$ & $P_{C H G}\left(R_{2} \mid S_{n}\right)$ \\
\hline S1 & 0.47 & 0.45 & 0.44 \\
\hline S2 & 0.53 & 0.55 & 0.56 \\
\hline
\end{tabular}

From table IV, V, VI class conditional probability of each factor for the relay node will be calculated as $\mathrm{P}_{\mathrm{SNR}}\left(\mathrm{R}_{1} \mid \mathrm{S}_{1}\right)=0.0004 /(0.0004+0.0007) \quad$ and $\mathrm{P}_{\mathrm{SNR}}\left(\mathrm{R}_{1} \mid \mathrm{S}_{2}\right)=0.0007 /(0.0004+0.0007)$, so that $\mathrm{P}_{\mathrm{SNR}}\left(\mathrm{R}_{1} \mid \mathrm{S}_{1}\right)=0.36$ and $\mathrm{P}_{\mathrm{SNR}}\left(\mathrm{R}_{1} \mid \mathrm{S}_{2}\right)=0.67$.

Similarly for $\mathrm{R}_{2}, \quad \mathrm{P}_{\mathrm{SNR}}\left(\mathrm{R}_{2} \mid \mathrm{S}_{1}\right)=0.0005 /$ $0.0005+0.0008$ and $P_{S N R}\left(R_{2} \mid S_{2}\right)=0.0008 /$ $(0.0005+0.0008)$ so that $P_{S N R}\left(R_{2} \mid S_{1}\right)=0.38$ and $P_{S N R}\left(R_{2} \mid S_{2}\right)=0.62$.

For CG prior probabilities will be given as $\mathrm{P}_{\mathrm{CG}}\left(\mathrm{R}_{1} \mid \mathrm{S}_{1}\right)=0.65 /(0.65+0.44) \quad$ and $\mathrm{P}_{\mathrm{CG}}\left(\mathrm{R}_{1} \mid \mathrm{S}_{2}\right)=0.44 /(0.65+0.44), \quad$ so $\quad$ that $\mathrm{P}_{\mathrm{CG}}\left(\mathrm{R}_{1} \mid \mathrm{S}_{1}\right)=0.6$ andP $_{\mathrm{CG}}\left(\mathrm{R}_{1} \mid \mathrm{S}_{2}\right)=0.4$.

Similarly, for $\mathrm{R}_{2}, \mathrm{P}_{\mathrm{CG}}\left(\mathrm{R}_{2} \mid \mathrm{S}_{1}\right)=0.56 / 0.56+$ 0.72 and $P_{C G}\left(R_{2} \mid S_{2}\right)=0.72 /(0.56+0.72)$ so that $\mathrm{P}_{\mathrm{CG}}\left(\mathrm{R}_{2} \mid \mathrm{S}_{1}\right)=0.44 \operatorname{andP}_{\mathrm{CG}}\left(\mathrm{R}_{2} \mid \mathrm{S}_{2}\right)=0.56$.

For CHG prior probabilities will be given as $P_{C H G}\left(R_{1} \mid S_{1}\right)=0.0005 /(0.0005+0.0006)$ and $\mathrm{P}_{\mathrm{CHG}}\left(\mathrm{R}_{1} \mid \mathrm{S}_{2}\right)=0.0006 /(0.0005+0.0006)$, so that $\mathrm{P}_{\mathrm{CHG}}\left(\mathrm{R}_{1} \mid \mathrm{S}_{1}\right)=0.45 \operatorname{andP}_{\mathrm{CHG}}\left(\mathrm{R}_{1} \mid \mathrm{S}_{2}\right)=0.55$.

Similarly, for $\quad \mathrm{R}_{2}, \mathrm{P}_{\mathrm{CHG}}\left(\mathrm{R}_{2} \mid \mathrm{S}_{1}\right)=0.0001 /$ $(0.0001+0.002)$ and $\quad \mathrm{P}_{\mathrm{CHG}}\left(\mathrm{R}_{2} \mid \mathrm{S}_{2}\right)=0.002 /$ $(0.0001+0.002)$, so that, $\mathrm{P}_{\mathrm{CHG}}\left(\mathrm{R}_{2} \mid \mathrm{S}_{1}\right)=$ 0.44 and $\mathrm{P}_{\mathrm{CHG}}\left(\mathrm{R}_{2} \mid \mathrm{S}_{2}\right)=0.56$. 
Table 6. Posterior Probabilities of SNR for Direct and Relay Path

\begin{tabular}{|c|c|c|}
\hline Sources & $\mathrm{P}_{\mathrm{SNR}}\left(\mathrm{S}_{\mathrm{n}} \mid \mathrm{R}_{1}\right)$ & $\mathrm{P}_{\mathrm{SNR}}\left(\mathrm{S}_{\mathrm{n}} \mid \mathrm{R}_{2}\right)$ \\
\hline $\mathrm{S} 1$ & 0.39 & 0.42 \\
\hline $\mathrm{S} 2$ & 0.61 & 0.58 \\
\hline
\end{tabular}

Table 7. Posterior Probabilities of CG for Direct and Relay Path

\begin{tabular}{|c|c|c|}
\hline Sources & $\mathrm{P}_{\mathrm{CG}}\left(\mathrm{S}_{\mathrm{n}} \mid \mathrm{R}_{1}\right)$ & $\mathrm{P}_{\mathrm{CG}}\left(\mathrm{S}_{\mathrm{n}} \mid \mathrm{R}_{2}\right)$ \\
\hline $\mathrm{S} 1$ & 0.62 & 0.47 \\
\hline $\mathrm{S} 2$ & 0.37 & 0.53 \\
\hline
\end{tabular}

Table 8. Posterior Probabilities of CHG for Direct and Relay Path

\begin{tabular}{|c|c|c|}
\hline Sources & $\mathrm{P}_{\mathrm{CHG}}\left(\mathrm{S}_{\mathrm{n}} \mid \mathrm{R}_{1}\right)$ & $\mathrm{P}_{\mathrm{CHG}}\left(\mathrm{S}_{\mathrm{n}} \mid \mathrm{R}_{2}\right)$ \\
\hline $\mathrm{S} 1$ & 0.42 & 0.41 \\
\hline $\mathrm{S} 2$ & 0.58 & 0.6 \\
\hline
\end{tabular}

From table 7, 8, and 9, the posterior probability of each factor for the relay node assignment will be calculated as $\mathrm{P}_{\mathrm{SNR}}\left(\mathrm{S}_{1} \mid \mathrm{R}_{1}\right)=(0.36)(0.54) /$ $((0.36)(0.54)+(0.67)(0.46))$ and $\mathrm{P}_{\mathrm{SNR}}\left(\mathrm{S}_{1} \mid \mathrm{R}_{2}\right)=$ $(0.38)(0.54) /((0.38)(0.54)+(0.62)(0.46))$, so that $\mathrm{P}_{\mathrm{SNR}}\left(\mathrm{S}_{1} \mid \mathrm{R}_{1}\right)=0.39$ and $\mathrm{P}_{\mathrm{SNR}}\left(\mathrm{S}_{1} \mid \mathrm{R}_{2}\right)=0.42$.

Similarly for $\mathrm{R}_{2}, \mathrm{P}_{\mathrm{SNR}}\left(\mathrm{S}_{2} \mid \mathrm{R}_{1}\right)=(0.67)(0.46) /$ $((0.67)(0.46)+(0.36)(0.54))$ and $\mathrm{P}_{\mathrm{SNR}}\left(\mathrm{S}_{2} \mid \mathrm{R}_{2}\right)=$ $(0.62)(0.46) /((0.62)(0.46)+(0.38)(0.54))$, so that $\mathrm{P}_{\mathrm{SNR}}\left(\mathrm{S}_{2} \mid \mathrm{R}_{1}\right)=0.61$ and $\mathrm{P}_{\mathrm{SNR}}\left(\mathrm{S}_{2} \mid \mathrm{R}_{2}\right)=0.58$.

For CG posterior probabilities will be given as $\mathrm{P}_{\mathrm{CG}}\left(\mathrm{S}_{1} \mid \mathrm{R}_{1}\right)=(0.6)(0.53) /((0.6)(0.53)+$ $(0.4)(0.47)) \quad \operatorname{andP}_{\mathrm{CG}}\left(\mathrm{S}_{1} \mid \mathrm{R}_{2}\right)=(0.44)(0.53) /$ $((0.44)(0.53)+(0.56)(0.47)), \quad$ so that $\mathrm{P}_{\mathrm{CG}}\left(\mathrm{S}_{1} \mid \mathrm{R}_{1}\right)=0.62$ andP $\mathrm{PG}_{\mathrm{CG}}\left(\mathrm{S}_{1} \mid \mathrm{R}_{2}\right)=0.47$.

Similarly for $\mathrm{R}_{2}, \quad \mathrm{P}_{\mathrm{CG}}\left(\mathrm{S}_{2} \mid \mathrm{R}_{1}\right)=(0.4)(0.47) /$ $((0.4)(0.47)+(0.6)(0.53))$ and $\mathrm{P}_{\mathrm{CG}}\left(\mathrm{S}_{2} \mid \mathrm{R}_{2}\right)=$ $(0.56)(0.47) /((0.56)(0.47)+(0.44)(0.53))$, so that $\mathrm{P}_{\mathrm{CG}}\left(\mathrm{S}_{2} \mid \mathrm{R}_{1}\right)=0.37$ and $\mathrm{P}_{\mathrm{CG}}\left(\mathrm{S}_{2} \mid \mathrm{R}_{2}\right)=0.53$.

For CHG posterior probabilities will be given as $\mathrm{P}_{\mathrm{CHG}}\left(\mathrm{S}_{1} \mid \mathrm{R}_{1}\right)=(0.45)(0.47) /((0.45)(0.47)+$ $(0.55)(0.53))$ and $\mathrm{P}_{\mathrm{CHG}}\left(\mathrm{S}_{1} \mid \mathrm{R}_{2}\right)=(0.44)(0.47) /$ $((0.44)(0.47)+(0.56)(0.53)), \quad$ so that $\mathrm{P}_{\mathrm{CHG}}\left(\mathrm{S}_{1} \mid \mathrm{R}_{1}\right)=0.42$ and $\mathrm{P}_{\mathrm{CHG}}\left(\mathrm{S}_{1} \mid \mathrm{R}_{2}\right)=0.41$.

Similarly for $\mathrm{R}_{2}, \mathrm{P}_{\mathrm{CHG}}\left(\mathrm{S}_{2} \mid \mathrm{R}_{1}\right)=(0.55)(0.53) /$ $((0.55)(0.53)+0.45)(0.47))$ and $\mathrm{P}_{\mathrm{CHG}}\left(\mathrm{S}_{2} \mid \mathrm{R}_{2}\right)=$ $(0.56)(0.53) /((0.56)(0.53)+(0.44)(0.47))$, so

that $\mathrm{P}_{\mathrm{CHG}}\left(\mathrm{S}_{2} \mid \mathrm{R}_{1}\right)=0.58$ and $\mathrm{P}_{\mathrm{CHG}}\left(\mathrm{S}_{2} \mid \mathrm{R}_{2}\right)=0.6$.

We calculated the mean of posterior probability for each factor of same path and select a relay node whose probability is better than the other relay node according to 20.In the above example $\mathrm{P}\left(\mathrm{S}_{1} \mid \mathrm{R}_{1}\right)>$ $P\left(\mathrm{~S}_{1} \mid \mathrm{R}_{2}\right)$ and $\mathrm{P}\left(\mathrm{S}_{2} \mid \mathrm{R}_{2}\right)>P\left(\mathrm{~S}_{2} \mid \mathrm{R}_{1}\right)$, so relay $\mathrm{R} 1$ is assigned to $S 1$ and relay $R 2$ is assigned to $S$.

\subsection{Simulation Results}

The results of simulation are obtained from the model shown in Figure 3 with comparison of BER vs SNR analysis. On the other hand, Figure 4 shows performance analysis of AS-BI scheme when relay $\mathrm{R} 1$ is selected for source S1 and relay R2 is selected for source S2 based on the posterior probability calculated through Bayes' formula.

In our simulation we considered four relay paths and two direct paths, such that two relay paths and one direct path is available for each source node. According to the simulation model direct path is between source and destination, while relay path is through relayR1 and relay R2. We transmitted $10^{6}$ symbol using BPSK modulation. At the destination MRC is implemented for the signal, which is received using direct path and through the relay which is selected. Results of the all the six paths are combined to show comparison.

In Figure 4 using AS-BI selection scheme we see reduction in BER of the selected relay R1 for source S1 in comparison with the rejected relay R2 for source S1 and selected relay R2 for source S2 in comparison with the rejected relay R1 for source S2. BER of relay path is also better than the direct path. Performance analysis shows improvement in decision making capability of relays due to which we got reduction in BER, and thus more efficient and reliable communication is achieved in terms of the better relay selection.

The simulation results are drawn in Figure 5 which depicts the comparison of BER vs SNR analysis. The performance analysis of AS-BI scheme is enhanced when relay $R 2$ is selected for source $S 1$ and relay $R 1$ is selected for source $S 2$ based on the posterior probability calculated through Bayes' formula.

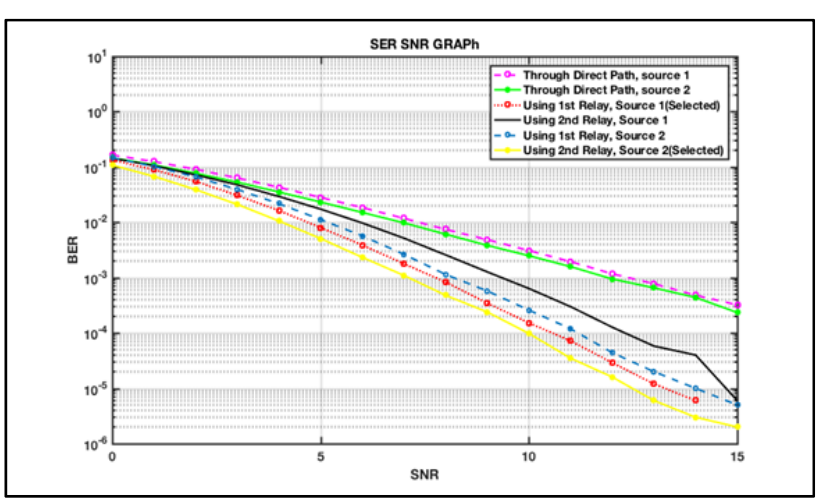

Figure 3. Performance analysis of AS-BI scheme when R1 is selected for $S 1$ and $R 2$ is selected for $S 2$ with BER reduction 


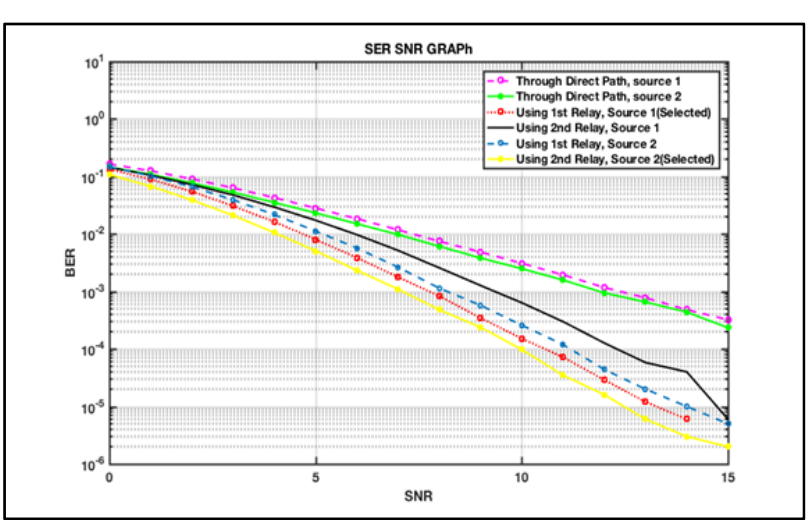

Figure 4. Performance analysis of AS-BI scheme when R2 is selected for $S 1$ and $R 1$ is selected for S2 with BER reduction

\section{Conclusion}

In this paper, the authors concentrated on the efficient way of relay assignment based on our proposed AS-BI scheme that adaptively select available relay nodes for cooperation based on their posterior probability. In terms of cooperative communication in cellular network relays are the user equipment and their selection efficiently, based on different channel conditions is the most critical thing. So, our aim was to use the relay nodes efficiently so that reliability of the network increases and thus improving the quality of the received signal by reducing error in it. This was done by considering three channel conditions SNR, CG, CHG as a prior knowledge for calculating the posterior probability and then selecting the best available relay towards the destination. We have presented the simulation results for the AS-BI scheme and given system model which shows performance analysis of BER vs SNR in terms of reduction in BER for fixed SNR thus verifying the proposed idea.

In the future we will expand this idea by considering more factors as a prior knowledge like relay power and its distance from the source which helps in more fine selection of the relay nodes.

\section{References}

[1]. Nosratinia, A., Hunter, T. E., \& Hedayat, A. (2004). Cooperative communication in wireless networks. IEEE communications Magazine, 42(10), 74-80.

[2]. Ferdouse, L., \& Anpalagan, A. (2015). Relay selection based on Bayesian decision theory in cooperative wireless networks. Canadian Journal of Electrical and Computer Engineering, 38(2), 116124.

[3]. Li, G. Y., Xu, Z., Xiong, C., Yang, C., Zhang, S., Chen, Y., \& Xu, S. (2011). Energy-efficient wireless communications: tutorial, survey, and open issues. IEEE Wireless communications, 18(6), 28-35.

[4]. Ke, F., Feng, S., \& Zhuang, H. (2010). Relay selection and power allocation for cooperative network based on energy pricing. IEEE Communications Letters, 14(5), 396-398.

[5]. Singh, S. P., \& Tyagi, A. (2016, December). Power allocation and relay selection for wireless relay networks. In 2016 International Conference on Signal Processing and Communication (ICSC) (pp. 134138). IEEE.

[6]. Wang, C. L., \& Chen, J. Y. (2015). Power allocation and relay selection for AF cooperative relay systems with imperfect channel estimation. IEEE Transactions on Vehicular Technology, 65(9), 7809-7813.

[7]. Xing, Y., Chen, Y., Lv, C., Gong, Z., \& Xu, L. (2016). Swarm intelligence-based power allocation and relay selection algorithm for wireless cooperative network. KSII Transactions on Internet and Information Systems (TIIS), 10(3), 1111-1130.

[8]. Siddiqi, S. J., Humayun, A., Rehman, H., Khan, I., \& Jan, S. (2013, September). Adaptive relay selection technique for cooperative network over Nakagami fading channels. In Eighth International Conference on Digital Information Management (ICDIM 2013) (pp. 161-65). IEEE.

[9]. Waqas, S. A., Jawad, M., Khan, I., Mahmood, M. A., Shah, I. A., \& Jan, S. (2013). Path (s) Finding and Selection Technique for Multi-Hop Mesh Cooperative Networks. Bahria University Journal of Information \& Communication Technology, 6(1), 50.

[10]. Qian, M., Liu, C., Fu, Y., \& Zhu, W. (2014). A Relay Selection and Power Allocation Scheme for Cooperative Wireless Sensor Networks. KSII Transactions on Internet and Information Systems (TIIS), 8(4), 1390-1405.

[11]. Li, Y., Liao, C., Wang, Y., \& Wang, C. (2015). Energy-efficient optimal relay selection in cooperative cellular networks based on double auction. IEEE Transactions on Wireless Communications, 14(8), 4093-4104.

[12]. Amarasuriya, G., Ardakani, M., \& Tellambura, C. (2010). Output-threshold multiple-relay-selection scheme for cooperative wireless networks. IEEE Transactions on Vehicular Technology, 59(6), 30913097. 
[13]. Sharma, S., Shi, Y., Hou, Y. T., \& Kompella, S. (2010). An optimal algorithm for relay node assignment in cooperative ad hoc networks. IEEE/ACM Transactions on networking, 19(3), 879-892.

[14]. Onat, F. A., Fan, Y., Yanikomeroglu, H., \& Poor, H. (2010). Threshold-based relay selection for detectand-forward relaying in cooperative wireless networks. EURASIP journal on wireless communications and networking, 2010(1), 1-9.

[15]. Viswanathan, R. (2007, January). Signal-to-noise ratio comparison of amplify-forward and direct link in wireless sensor networks. In 2007 2nd International Conference on Communication Systems Software and Middleware (pp. 1-4). IEEE.

[16]. Yang, W., Li, L., Wu, G., Wang, H., \& Wang, Y. (2010, September). Joint uplink and downlink relay selection in cooperative cellular networks. In 2010 IEEE 72nd Vehicular Technology ConferenceFall (pp. 1-5). IEEE.

[17]. Michalopoulos, D. S., \& Karagiannidis, G. K. (2008). Performance analysis of single relay selection in Rayleigh fading. IEEE Transactions on Wireless Communications, 7(10), 3718-3724.

[18]. Zarin, N., Mahmud, S. A., \& Khan, I. (2012, December). Relay based cooperative spectrum sensing in cognitive radio networks over rayleigh fading channel with path loss effects. In 2012 15th International Multitopic Conference (INMIC)(pp. 291-296). IEEE.

[19]. Wang, B., Han, Z., \& Liu, K. R. (2008). Distributed relay selection and power control for multiuser cooperative communication networks using Stackelberg game. IEEE Transactions on Mobile Computing, 8(7), 975-990.

[20]. Su, W., Sadek, A. K., \& Liu, K. R. (2008). Cooperative communication protocols in wireless networks: performance analysis and optimum power allocation. Wireless Personal Communications, 44(2), 181-217.

[21]. Luo, J., Blum, R. S., Greenstein, L. J., Cimini, L. J., \& Haimovich, A. M. (2004, September). New approaches for cooperative use of multiple antennas in ad hoc wireless networks. In IEEE 60th Vehicular Technology Conference, 2004. VTC2004-Fall. 2004 (Vol. 4, pp. 2769-2773). IEEE.

[22]. Yang, D., Fang, X., \& Xue, G. (2011, June). OPRA: Optimal relay assignment for capacity maximization in cooperative networks. In 2011 IEEE International Conference on Communications (ICC) (pp. 1-6). IEEE.

[23]. Xu, H., Huang, L., Wang, G., Xu, T., \& Liu, G. (2010). Joint relay assignment and power allocation for cooperative communications. Wireless Networks, 16(8), 2209-2219.
[24]. Ng, T. C. Y., \& Yu, W. (2007). Joint optimization of relay strategies and resource allocations in cooperative cellular networks. IEEE Journal on Selected areas in Communications, 25(2), 328-339.

[25]. Fares, S. A., Adachi, F., \& Kudoh, E. (2008, August). Novel cooperative relaying network scheme with exchange communication and distributed transmit beamforming. In The 5th Ieee Vts Asia Pacific Wireless Communications Symposiums (APWSC 2008), Sendai, Japan, August.

[26]. Zhang, H., Hong, P., \& Xue, K. (2013). Mobilebased relay selection schemes for multi-hop cellular networks. Journal of Communications and Networks, 15(1), 45-53.

[27]. Bletsas, A., Khisti, A., Reed, D. P., \& Lippman, A. (2006). A simple cooperative diversity method based on network path selection. IEEE Journal on selected areas in communications, 24(3), 659-672.

[28]. Xie, W., Chen, H., \& Wu, Z. (2009, December). Relay selection schemes for the trade-off between cell lifetime and system capacity. In IET International Communication Conference on Wireless Mobile and Computing (CCWMC 2009) (pp. 526-529). IET.

[29]. Sheng, Z., Fan, J., Liu, C. H., Leung, V. C., Liu, X., \& Leung, K. K. (2014). Energy-efficient relay selection for cooperative relaying in wireless multimedia networks. IEEE Transactions on Vehicular Technology, 64(3), 1156-1170.

[30]. Kadloor, S., \& Adve, R. (2010). Relay selection and power allocation in cooperative cellular networks. IEEE Transactions on Wireless Communications, 9(5), 1676-1685.

[31]. Chen, G., Tian, Z., Gong, Y., Chen, Z., \& Chambers, J. A. (2014). Max-ratio relay selection in secure buffer-aided cooperative wireless networks. IEEE transactions on information forensics and security, 9(4), 719-729.

[32]. Gunantara, N., \& Hendrantoro, G. (2013). MultiObjective Cross-Layer Optimization for Selection of Cooperative Path Pairs in Multihop Wireless Ad hoc Networks. Journal of Communications Software and Systems, 9(3), 170-177.

[33]. Bletsas, A., Lippnian, A., \& Reed, D. P. (2005, May). A simple distributed method for relay selection in cooperative diversity wireless networks, based on reciprocity and channel measurements. In 2005 IEEE 61st Vehicular Technology Conference (Vol. 3, pp. 1484-1488). IEEE.

[34]. Wang, B., Han, Z., \& Liu, K. R. (2008). Distributed relay selection and power control for multiuser cooperative communication networks using Stackelberg game. IEEE Transactions on Mobile Computing, 8(7), 975-990. 